04

\title{
Влияние температуры на ионизационный коэффициент и напряжение зажигания таунсендовского разряда в смеси аргона с парами ртути
}

\author{
(С) Г.Г. Бондаренко, ${ }^{1}$ М.Р. Фишер, ${ }^{2}$ В.И. Кристя ${ }^{2, \text { 甲 }}$ \\ ${ }^{1}$ Национальный исследовательский университет „Высшая школа экономики“, \\ 101000 Москва, Россия \\ ${ }^{2}$ Московский государственный технический университет им. Н.Э. Баумана, Калужский филиал, \\ 248000 Калуга, Россия \\ ฯ e-mail: kristya@bmstu-kaluga.ru
}

(Поступило в Редакцию 7 июня 2016 г.)

В широком интервале значений приведенной напряженности электрического поля и температуры проведено моделирование кинетики основных типов заряженных и возбужденных частиц в слаботочном разряде в смеси аргон-ртуть, используемой в газоразрядных осветительных лампах. Найдены температурные зависимости вкладов различных механизмов образования и потерь ионов и метастабильных атомов в поддержание их баланса. Показано, что на этапе зажигания разряда в лампе, когда содержание ртути в смеси мало́, возрастание ионизационного коэффициента в ней по сравнению с чистым аргоном практически полностью обусловлено реакцией Пеннинга, роль которой увеличивается с уменьшением приведенной напряженности электрического поля в межэлектродном промежутке. Рассчитаны зависимости напряжения зажигания разряда от межэлектродного расстояния (кривые Пашена) при различных температурах смеси и дано объяснение немонотонной зависимости его величины от температуры.

DOI: 10.21883/JTF.2017.02.44125.1918

\section{Введение}

В настоящее время, несмотря на интенсивную разработку светодиодных технологий, одним из основных типов осветительных приборов остаются газоразрядные лампы [1-3]. Часто в них в качестве рабочего газа используется смесь аргона с концентрацией, соответствующей его давлению $10^{2}-10^{4}$ Ра при комнатной температуре, и паров ртути, концентрация которых зависит от температуры. При включении лампы происходит пробой газа в межэлектродном промежутке под действием напряжения, приложенного к ее электродам, и зажигается слаботочный (таунсендовский) разряд, переходящий затем в тлеющий разряд с катодным падением напряжения в несколько сот вольт. В результате бомбардировки катода ионами, ускоряемыми в катодном слое тлеющего разряда, с его поверхности происходит эмиссия электронов, необходимых для поддержания разряда, а также его нагрев и распыление эмиссионного вещества. Когда температура катода становится достаточно высокой, с него начинается термическая эмиссия электронов, и разряд переходит в дуговой, характеризующийся значительно меньшим катодным падением напряжения, чем тлеющий разряд [4-6]. При этом наиболее интенсивное распыление катода, приводящее к потере эмиссионного вещества и ограничивающее срок службы лампы, происходит до перехода разряда в дуговую форму, так как долговечность лампы в непрерывном режиме работы значительно превосходит ее величину в режиме периодических включений и выключений $[7,8]$.

Напряжение пробоя, так же как и напряжение горения тлеющего разряда, от которого существенно зависят энергии бомбардирующих катод ионов, определяется процессами эмиссии электронов с катода и ионизации рабочего газа в разрядном объеме. Первый из них характеризуется эффективным коэффициентом ионноэлектронной эмиссии, равным среднему числу эмиттируемых электронов в расчете на один падающий на катод ион, а второй - ионизационным коэффициентом (ИК), равным среднему числу ионизаций атомов рабочего газа на единице длины разряда в расчете на один электрон. Особенность смеси аргона с парами ртути состоит в том, что в ней, кроме прямой ионизации атомов электронами, происходит также ионизация атомов ртути при столкновениях с метастабильными возбужденными атомами аргона (реакция Пеннинга) $[9,10]$. Это обусловливает зависимость величины ИК и напряжения зажигания разряда от температуры, так как при ее возрастании увеличивается содержание ртути в смеси. Поэтому исследование процессов, происходящих при протекании электрического тока в смеси аргон-ртуть, представляет значительный интерес. Однако данному вопросу посвящено лишь небольшое число работ. В частности, в $[11,12]$ измерены значения ИК в слаботочном разряде между плоскими электродами при различных значениях относительного содержания ртути в смеси и напряженности электрического поля в межэлектродном промежутке. В работе [13] проведены расчеты зависимости ИК и вкладов в него нескольких типов межчастичных взаимодействий от приведенной напряженности электрического поля. При этом величина концентрации электронов выбиралась из условия соответствия результатов расчета экспериментальным данным работы [11], а концентрации ионов и возбужденных атомов определя- 
лись из усредненных по объему уравнений их баланса. Роль различных взаимодействий в смеси аргон-ртуть детально исследована лишь в положительном столбе дугового разряда [14-18], для которого характерны малые значения напряженности электрического поля и средней энергии электронов. В [19] проведено численное моделирование динамики пробоя в колбе лампы и исследованы протекающие в ней процессы при нескольких фиксированных величинах относительного содержания ртути в смеси. В ряде работ, например в $[10,12]$, экспериментально установлено, что зависимость напряжения зажигания разряда в такой смеси от ее температуры является немонотонной: возрастающей при температурах порядка комнатной и убывающей при ее более высоких значениях, наблюдающихся в течение некоторого промежутка времени после погасания дугового разряда в лампе. Детальное же изучение влияния различных типов межчастичных взаимодействий на характеристики разряда в смеси аргон-ртуть на этапе его зажигания при различных температурах до настоящего времени не проводилось.

В настоящей работе рассчитан перенос электронов, ионов и метастабильных возбужденных атомов в слаботочном разряде в смеси аргон-ртуть в достаточно широком интервале значений приведенной напряженности электрического поля и температуры. Определены концентрации заряженных и возбужденных частиц в разрядном промежутке, вклады различных типов межчастичных взаимодействий в поддержание их баланса как функции относительного содержания ртути в смеси, а также вычислено напряжение зажигания разряда при различных температурах рабочего газа.

\section{Описание модели разряда}

Пусть между параллельными плоскими электродами, расстояние $d$ между которыми много меньше их поперечных размеров, приложено напряжение $U$, достаточное для пробоя межэлектродного промежутка, заполненного аргоном с концентрацией $n_{\mathrm{Ar}}$ и насыщенными парами ртути с концентрацией $n_{\mathrm{Hg}}$. Ось $z$ координатной системы считается направленной перпендикулярно к электродам, причем поверхность катода совпадает с плоскостью $z=0$, а анода - с плоскостью $z=d$. На начальном этапе разряда плотность тока $j$ в нем достаточно мала, поэтому объемный заряд не искажает распределения электрического поля в межэлектродном промежутке, которое во всех точках направлено параллельно оси $z$ и равно $E=U / d$, т.е. разряд является слаботочным [5,6].

Под действием поля электроны, образующиеся при ионизации атомов, ускоряются в направлении анода, а ионы - в направлении катода, сталкиваясь при этом с нейтральными атомами. Для моделирования движения электронов в настоящей работе применяется методика, основанная на использовании метода Монте-Кар- ло [20-22]. Учитывается упругое рассеяние электронов на атомах компонент смеси, а также возбуждение и ионизация ими невозбужденных и метастабильных атомов с использованием зависимостей сечений этих процессов от скорости электрона, приведенных в [23-25]. В процессе расчета траекторий первичных и вторичных электронов (эмитируемых с катода и образующихся в разрядном промежутке при ионизации атомов компонент смеси) формируется функция их распределения по скоростям $f_{e}\left(z_{i}, v, v_{z}\right)$ в каждом из $s$-интервалов длины $\Delta z=d / s$, на которые разбивается межэлектродный промежуток, где $z_{i}=(i-0.5) \Delta z, i=1, \ldots, s, v$ и $v_{z}$ скорость электрона и ее продольная составляющая.

Основными типами ионов в слаботочном разряде в смеси аргона с парами ртути являются атомарные и молекулярные ионы обоих ее компонент $\mathrm{Ar}^{+}, \mathrm{Ar}_{2}^{+}$и $\mathrm{Hg}^{+}$, $\mathrm{Hg}_{2}^{+}[19,26]$, а из возбужденных атомов наибольшую концентрацию имеют метастабили аргона $4^{3} P_{0}, 4^{3} P_{2}$ и ртути $6^{3} P_{0}, 6^{3} P_{2}$. Так как энергетические уровни двух типов метастабилей каждого газа достаточно близки, их можно рассматривать как идентичные, обозначив через $\mathrm{Ar}^{*}$ и $\mathrm{Hg}^{*}$ соответственно [13,19,27]. Атомы же, возбуждаемые на резонансные уровни (они обозначаются символами $\mathrm{Ar}^{* *}$ и $\left.\mathrm{Hg}^{* *}\right)$, быстро девозбуждаются, поэтому они не рассматриваются в данной модели. Учитываемые типы ион-атомных и атом-атомных взаимодействий, а также значения констант соответствующих процессов и источники, из которых взяты их значения, приведены в таблице.

При достаточно большой напряженности электрического поля в разрядном промежутке, характерной для слаботочного разряда, движение атомарных и молекулярных ионов в нем с учетом взаимодействий, указанных в таблице, может быть описано на основе макроскопических уравнений их переноса, а метастабилей - на основе соответствующих диффузионных уравнений [35-37]. После их решения методом конечных разностей вычисляется количество электронов $\Delta n_{e}$, образующихся в единицу времени в единице разрядного объема при столкновениях тяжелых частиц (с учетом их потерь в процессах рекомбинации), и находятся соответствующие количества вторичных электронов, которые необходимо добавить в ячейки длиной $\Delta z$, на которые разбивается межэлектродный промежуток, при моделировании кинетики электронов методом Монте-Карло.

Затем снова производится моделирование движения электронов в разрядном промежутке с учетом дополнительных вторичных электронов и опять рассчитывается перенос ионов и метастабилей. Такой цикл повторяется пока относительная разность значений величин в последовательных итерациях станет достаточно малой.

После нахождения функции распределения электронов по скоростям $f_{e}\left(z, v, v_{z}\right)$ может быть вычислен ИК смеси как сумма слагаемых, учитывающих вклады в него прямой ионизации атомов аргона и ртути электронами 
Учитываемые в модели ион-атомные и атом-атомные взаимодействия, а также значения их констант

\begin{tabular}{c|c|c}
\hline Реакция & Константа & Источник \\
\hline $\mathrm{Ar}^{+}+2 \mathrm{Ar} \rightarrow \mathrm{Ar}_{2}^{+}+\mathrm{Ar}$ & $2.7 \cdot 10^{-43} \mathrm{~m}^{6} \mathrm{~s}^{-1}$ & {$[27]$} \\
$\mathrm{Hg}^{+}+2 \mathrm{Hg} \rightarrow \mathrm{Hg}_{2}^{+}+\mathrm{Hg}$ & $1.0 \cdot 10^{-43} \mathrm{~m}^{6} \mathrm{~s}^{-1}$ & {$[16]$} \\
$\mathrm{Ar}^{*}+\mathrm{Ar}^{*} \rightarrow \mathrm{Ar}_{2}^{+}+e$ & $5.7 \cdot 10^{-16} \mathrm{~m}^{3} \mathrm{~s}^{-1}$ & {$[27]$} \\
$\mathrm{Ar}^{*}+\mathrm{Ar}^{*} \rightarrow \mathrm{Ar}^{+}+\mathrm{Ar}+e$ & $6.2 \cdot 10^{-16} \mathrm{~m}^{3} \mathrm{~s}^{-1}$ & {$[28]$} \\
$\mathrm{Hg}^{*}+\mathrm{Hg}^{*} \rightarrow \mathrm{Hg}_{2}^{+}+e$ & $1.0 \cdot 10^{-20} v_{a} \mathrm{~m}^{3} \mathrm{~s}^{-1}$ & {$[29]$} \\
$\mathrm{Hg}^{*}+\mathrm{Hg}^{*} \rightarrow \mathrm{Hg}^{+}+\mathrm{Hg}+e$ & $2.4 \cdot 10^{-19} v_{a} \mathrm{~m}^{3} \mathrm{~s}^{-1}$ & {$[15]$} \\
$\mathrm{Ar}^{*}+\mathrm{Hg} \rightarrow \mathrm{Ar}+\mathrm{Hg}^{+}+e$ & $9.0 \cdot 10^{-16} \mathrm{~m}^{3} \mathrm{~s}^{-1}$ & {$[30]$} \\
$\mathrm{Ar}^{+}+\mathrm{Hg} \rightarrow \mathrm{Ar}+\mathrm{Hg}^{+}$ & $1.5 \cdot 10^{-17} \mathrm{~m}^{3} \mathrm{~s}^{-1}$ & {$[28]$} \\
$\mathrm{Ar}+\mathrm{Ar} \rightarrow 2 \mathrm{Ar}$ & $3.0 \cdot 10^{-21} \mathrm{~m}^{3} \mathrm{~s}^{-1}$ & {$[31]$} \\
$\mathrm{Hg}+\mathrm{Hg} \rightarrow 2 \mathrm{Hg}$ & $8.0 \cdot 10^{-21} v_{a} \mathrm{~m}^{3} \mathrm{~s}^{-1}$ & {$[32]$} \\
$\mathrm{Hg}^{*}+\mathrm{Ar} \rightarrow \mathrm{Hg}^{* *}+\mathrm{Ar}$ & $2.0 \cdot 10^{-24} v_{a} \mathrm{~m}^{3} \mathrm{~s}^{-1}$ & {$[28]$} \\
$\mathrm{Ar}^{*}+2 \mathrm{Ar} \rightarrow \mathrm{Ar}_{2}+\mathrm{Ar}$ & $1.1 \cdot 10^{-43} \mathrm{~m}^{6} \mathrm{~s}^{-1}$ & {$[33]$} \\
$\mathrm{Hg}^{*}+2 \mathrm{Hg} \rightarrow \mathrm{Hg}_{2}+\mathrm{Hg}$ & $1.6 \cdot 10^{-43} \mathrm{~m}^{6} \mathrm{~s}^{-1}$ & {$[26]$} \\
$\mathrm{Ar}^{+}+e \rightarrow \mathrm{Ar}^{* *}+h v$ & $1.0 \cdot 10^{-17} \mathrm{~m}^{3} \mathrm{~s}^{-1}$ & {$[34]$} \\
$\mathrm{Hg}^{+}+e \rightarrow \mathrm{Hg}^{* *}+h v$ & $1.0 \cdot 10^{-14} \mathrm{~m}^{3} \mathrm{~s}^{-1}$ & {$[27]$} \\
$\mathrm{Ar}_{2}^{+}+e \rightarrow \mathrm{Ar}^{* *}+\mathrm{Ar}$ & $8.5 \cdot 10^{-13}\left(T / T_{e}\right)^{0.67} \mathrm{~m}^{3} \mathrm{~s}^{-1}$ & {$[16]$}
\end{tabular}

Примечание. $v_{a}-$ средняя относительная скорость взаимодействующих атомов; $T_{e}, \mathrm{~K}-$ электронная температура; $T, \mathrm{~K}-$ температура смеси.

и ионизации при столкновениях тяжелых частиц:

$$
\alpha(z)=\alpha_{\mathrm{Ar}}(z)+\alpha_{\mathrm{Hg}}(z)+\alpha_{P e n}(z),
$$

где

$$
\begin{gathered}
\alpha_{\mathrm{Ar}}(z)=n_{\mathrm{Ar}} \iint \sigma_{i \operatorname{Ar}}(v) f_{e}\left(z, v, v_{z}\right) v d v d v_{z} / J_{e}(z) \\
\alpha_{\mathrm{Hg}}(z)=n_{\mathrm{Hg}} \iint \sigma_{i \mathrm{Hg}}(v) f_{e}\left(z, v, v_{z}\right) v d v d v_{z} / J_{e}(z), \\
\alpha_{P e n}(z)=\Delta n_{e} / J_{e}(z) \\
J_{e}(z)=\iint f_{e}\left(z, v, v_{z}\right) v_{z} d v d v_{z}
\end{gathered}
$$

$\sigma_{i \operatorname{Ar}}(v)$ и $\sigma_{i \mathrm{Hg}}(v)$ - сечения ионизации атомов аргона и ртути электроном.

Зависимость ИК $\alpha$ и его компонент, так же как и других характеристик разряда, от координаты $z$ обусловлена тем, что вследствие наличия краевых эффектов вблизи электродов распределение электронов по скоростям близко к равновесному при заданной напряженности электрического поля $E$ лишь в центральной части разрядного промежутка, где эти величины достигают своих установившихся значений [20,38].

\section{Результаты расчетов и их обсуждение}

Вычисления проводились для разрядного промежутка длиной $d=1.5 \cdot 10^{-3} \mathrm{~m}$, заполненного чистыми аргоном и ртутью, а также смесью аргона с концентрацией $n_{\mathrm{Ar}}=6.57 \cdot 10^{23} \mathrm{~m}^{-3}$, соответствующей его давлению 2660 Ра при температуре $20^{\circ} \mathrm{C}$, и насыщенных паров ртути, концентрация которых быстро растет с увеличением температуры [39], так что их относительная концентрация $n_{\mathrm{Hg}} / n$ (где $\left.n=n_{\mathrm{Ar}}+n_{\mathrm{Hg}}\right)$ в температурном интервале от $-30^{\circ} \mathrm{C}$ до $+130^{\circ} \mathrm{C}$ изменяется от $5 \cdot 10^{-7}$ до $5 \cdot 10^{-2}$ (рис. 1). Использовалось значение плотности разрядного тока $j=1 \cdot 10^{-5} \mathrm{~A} \cdot \mathrm{m}^{-2}$, для которой при $U \sim 10^{2} \mathrm{~V}$ разряд является слаботочным [5].

В процессе моделирования движения электронов в разряде количество эмиттируемых с катода первичных электронов выбиралось равным $10^{3}-10^{4}$ и предполагалось, что их начальные энергии равномерно распределены в интервале от 0 до $4 \mathrm{eV}$, а направления вылета изотропно распределены по углу относительно нормали к поверхности. Разрядный промежуток, а также интервалы изменения $v$ и $v_{z}$ разбивались на 100 частичных интервалов, т.е. $s=100$. При расчете переноса ионов и метастабилей в смеси вследствие малого содержания в ней ртути использовались значения подвижностей в аргоне атомарных и молекулярных ионов аргона и ртути из $[27,28,40]$, а также значения коэффициентов диффузии в аргоне метастабильных атомов обоих газов, приведенные в [28,32].

На рис. 2 представлены полученные зависимости величины ИК в центре разрядного промежутка (при $z=d / 2$ ) от приведенной напряженности электрического поля $E / n$ в чистых аргоне и ртути, в смеси аргонртуть при $n_{\mathrm{Hg}} / n=6 \cdot 10^{-3}$, а также его экспериментальные значения $[13,41,42]$. Видно, что имеет место соответствие вычисленных и измеренных значений $\alpha$, подтверждающее удовлетворительную точность использованной модели. Найденная зависимость ИК в смеси аргон-ртуть от относительного содержания ртути в ней (рис. 3) также согласуется с экспериментальным данными, причем максимальное значение ИК достигается при $n_{\mathrm{Hg}} / n \approx 5 \cdot 10^{-3}$. В случае значения концентрации аргона в смеси $n_{\mathrm{Ar}}=6.57 \cdot 10^{23} \mathrm{~m}^{-3}$, характерного для 


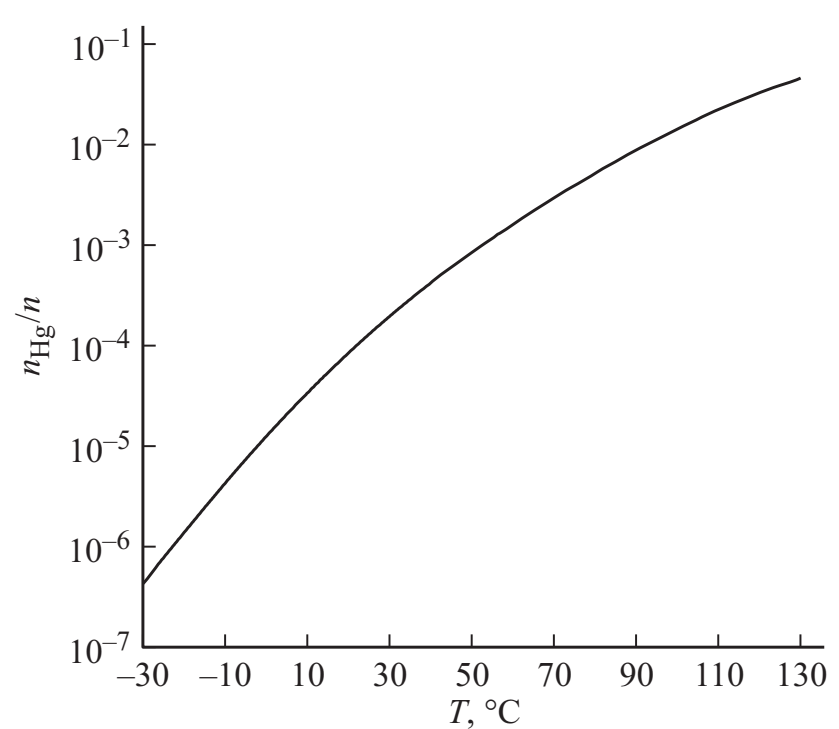

Рис. 1. Зависимость относительной концентрации насыщенных паров ртути в смеси аргон-ртуть от температуры при $n_{\mathrm{Ar}}=6.57 \cdot 10^{23} \mathrm{~m}^{-3}[39]$.

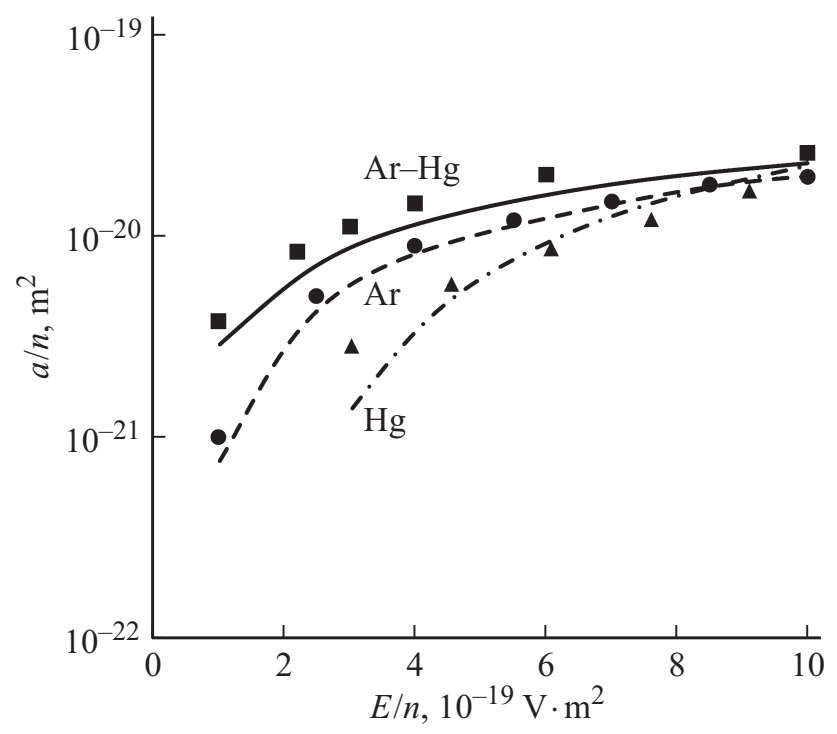

Рис. 2. Зависимость ИК от приведенной напряженности электрического поля в аргоне (штриховая линия), ртути (штрихпунктирная линия) и их смеси при $n_{\mathrm{Hg}} / n=6 \cdot 10^{-3}$ (сплошная линия). Символы $\bullet, \mathbf{\Delta}, \mathbf{\square}-$ экспериментальные значения ИК для аргона [41], ртути [42] и их смеси [13] соответственно.

дуговых ртутных ламп высокого давления [4,12], это имеет место при температуре около $70^{\circ} \mathrm{C}$, т. е. на этапе зажигания лампы интенсивность процесса ионизации возрастает с увеличением температуры окружающей среды. При этом основной вклад в величину $\Delta n_{e}$, определяющую увеличение ИК в смеси по сравнению с чистым аргоном, дает слагаемое, обусловленное пеннинговской ионизацией атомов ртути метастабилями аргона, роль которой становится более существенной с уменьшением напряженности электрического поля $E$. Вклад же прямой ионизации атомов ртути электронами ввиду их малой относительной концентрации, как видно из рис. 3, при $n_{\mathrm{Hg}} / n<10^{-2}$ незначителен.

Рассчитанные зависимости концентраций ионов и метастабильных атомов в центре разрядного промежутка от относительного содержания ртути в смеси приведены на рис. 4. Из него следует, что в рассмотренном интервале значений $n_{\mathrm{Hg}} / n$ основными типами частиц в разряде, кроме электронов, являются атомарные ионы и метастабильные возбужденные атомы компонент смеси, а концентрации молекулярных ионов имеют по крайней

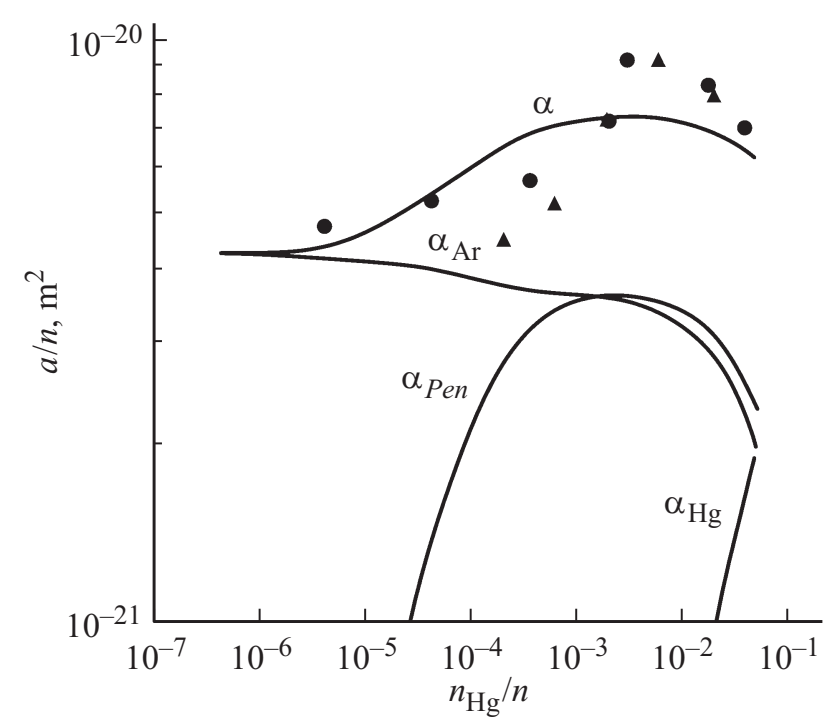

Рис. 3. Зависимость ИК и его компонент от относительной концентрации ртути в смеси при $E / n=2.5 \cdot 10^{-19} \mathrm{~V} \cdot \mathrm{m}^{2}$. Линии - результаты расчета, символы •, $\mathbf{\Delta}-$ экспериментальные значения ИК $[12,13]$.

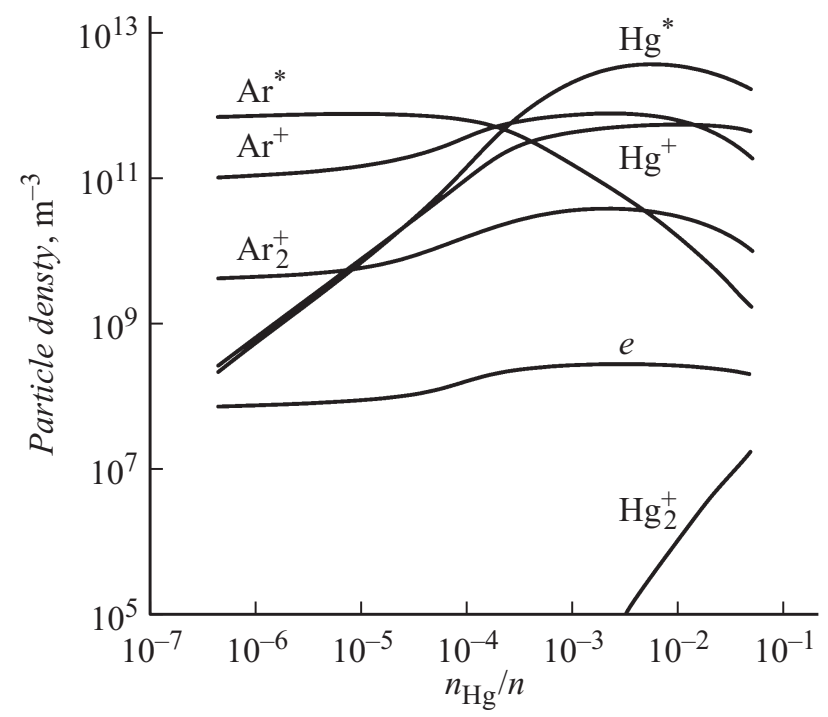

Рис. 4. Концентрации основных типов заряженных и возбужденных частиц в центре разрядного промежутка как функции относительного содержания ртути в смеси при $E / n=2.5 \cdot 10^{-19} \mathrm{~V} \cdot \mathrm{m}^{2}$. 

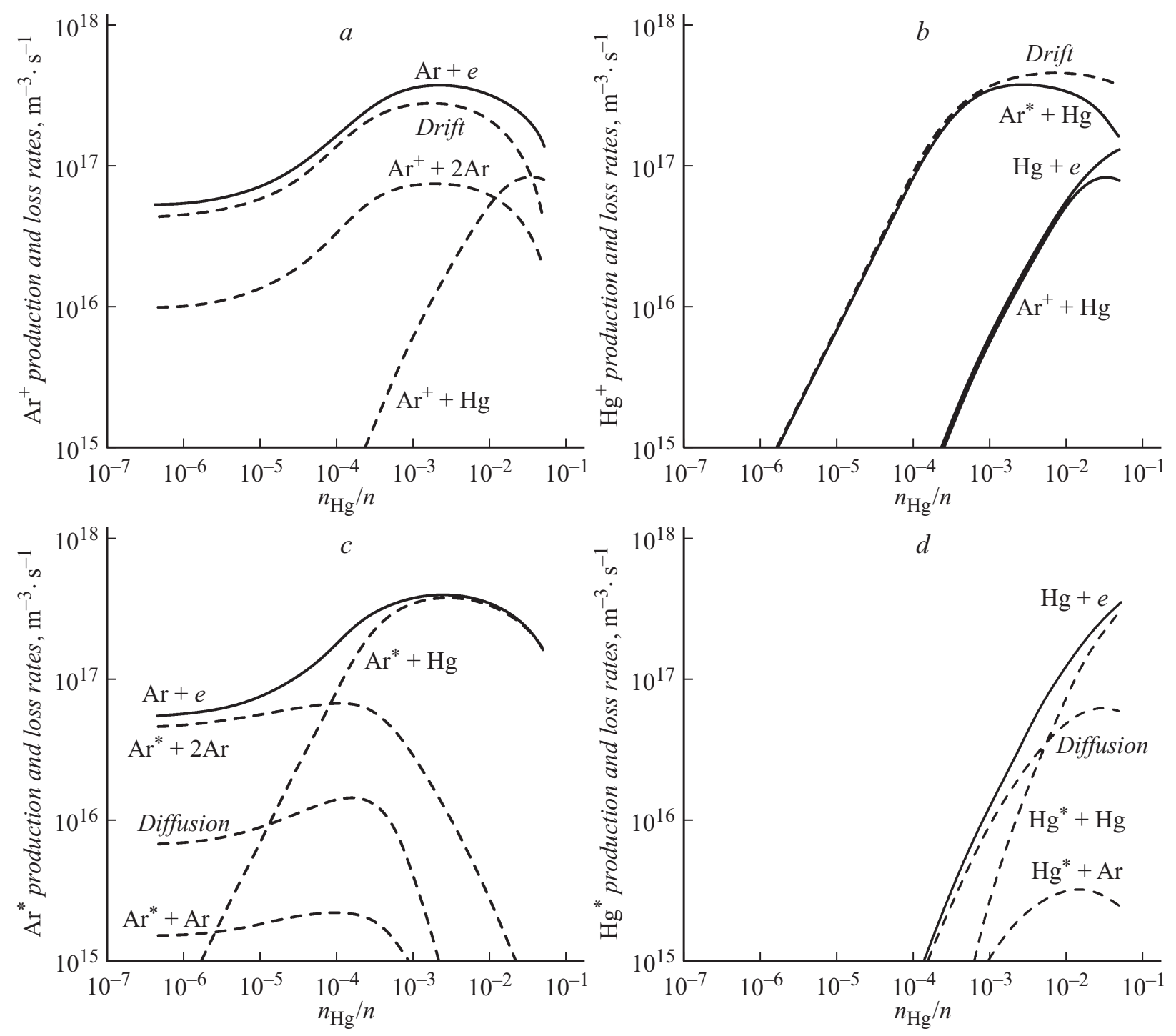

Рис. 5. Вклады основных механизмов образования (сплошные линии) и потерь (штриховые линии) атомарных ионов аргона (a) и ртути $(b)$, а также метастабилей аргона $(c)$ и ртути $(d)$ как функции относительного содержания ртути в смеси при $E / n=2.5 \cdot 10^{-19} \mathrm{~V} \cdot \mathrm{m}^{2}$.

мере на порядок меньшую величину (что согласуется с результатами работы [19]). При этом, как видно из рис. 5, на котором представлены усредненные по разрядному промежутку вклады различных механизмов их образования и потерь как функции $n_{\mathrm{Hg}} / n$, атомарные ионы аргона $\mathrm{Ar}^{+}$возникают при ионизации атомов аргона электронами, в то время как основной источник атомарных ионов ртути $\mathrm{Hg}^{+}$при $n_{\mathrm{Hg}} / n<10^{-2}-$ пеннинговская ионизация атомов ртути метастабилями аргона, а при бо́льших величинах $n_{\mathrm{Hg}} / n$ существенную роль играет также их прямая ионизация электронами и нерезонансная перезарядка ионов аргона на атомах ртути. Потери обоих типов ионов обусловлены их дрейфом к катоду под действием электрического поля, а для атомарных ионов аргона заметную роль играет также их конверсия в молекулярные ионы. Метастабили аргона $\mathrm{Ar}^{*}$ возникают при возбуждении атомов аргона электронами и разрушаются при $n_{\mathrm{Hg}} / n<10^{-4}$ главным образом в результате их тушения при столкновениях с невозбужденными атомами аргона, а при большем содержании ртути в смеси - в процессе пеннинговской ионизации ее атомов. Метастабили же ртути $\mathrm{Hg}^{*}$ образуются в результате возбуждения атомов ртути электронами и при $n_{\mathrm{Hg}} / n<10^{-2}$ диффундируют к электродам и девозбуждаются при столкновении с их поверхностью, а при $n_{\mathrm{Hg}} / n>10^{-2}$ основным механизмом их разрушения является тушение при столкновениях с невозбужденными атомами ртути. Роль всех других межчастичных взаимодействий в рассмотренном интервале значений параметров разряда незначительна.

Из рис. 1-5 следует, что при возрастании температуры смеси $T$ до $70^{\circ} \mathrm{C}$, обусловливающем увеличение относительной концентрации $n_{\mathrm{Hg}} / n$ ртути в смеси до величины $5 \cdot 10^{-3}$, растет интенсивность пеннинговской 


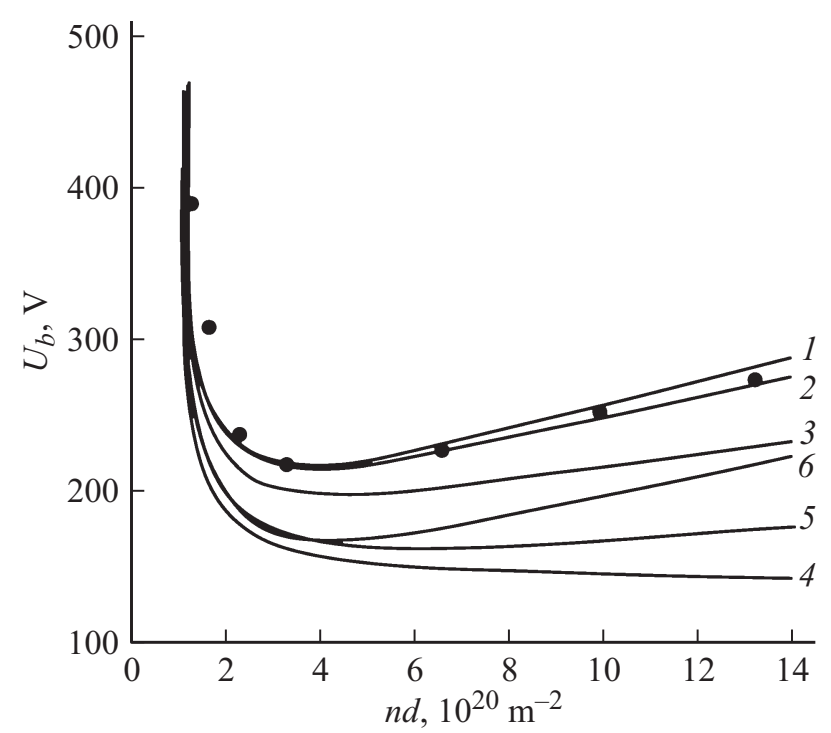

Рис. 6. Кривые Пашена в аргоне (1) и в смеси аргон-ртуть при температуре -10 (2), 10 (3), 70 (4), 90 (5), $130^{\circ} \mathrm{C}(6)$. Точки - экспериментальные значения $U_{b}$ для разряда в аргоне [43].

ионизации атомов ртути метастабилями аргона и увеличивается концентрация электронов, а также плотность их тока. В результате увеличиваются интенсивности процессов ионизации атомов аргона и ртути и возбуждения атомов аргона на метастабильные уровни электронами, а следовательно, и интенсивность пеннинговской ионизации атомов ртути метастабилями аргона. При этом происходит рост концентрации ионов и метастабилей ртути, а также увеличение ИК смеси, в то время как концентрация метастабилей аргона снижается. В случае же более высокой температуры смеси, превосходящей $70^{\circ} \mathrm{C}$, при ее увеличении вследствие роста концентрации ртути происходит существенное увеличение потерь энергии электронов при столкновениях с ее атомами. В результате уменьшается доля электронов, имеющих скорости, достаточные для возбуждения атомов аргона (энергетическое распределение электронов сужается [19]). Это приводит к снижению интенсивности процессов ионизации атомов аргона электронами и образованию метастабилей аргона, обусловливающему уменьшение ИК смеси.

С использованием рассчитанных значений ИК в разрядном промежутке можно найти напряжение зажигания разряда между электродами $U_{b}$ из условия его поддержания $[5,6]$

$$
\int_{0}^{d} \alpha\left(z, U_{b} / d\right) d z=\ln \left(1+1 / \gamma_{\mathrm{eff}}\right),
$$

где $\gamma_{\text {eff }}$ - эффективный коэффициент ионно-электронной эмиссии катода.

Вычисленные из (2) зависимости $U_{b}$ от $p d$, где $p-$ давление газовой смеси, (кривые Пашена) для разряда в аргоне и его смеси с парами ртути при различных температурах приведены на рис. 6 (значение $\gamma_{\mathrm{eff}}=0.0125$ выбрано из условия совпадения вычисленной величины $U_{b}$ в точке минимума кривой с его экспериментальным значением для разряда в чистом аргоне, найденным в [43]). В случае разряда в аргоне эта зависимость хорошо согласуется с экспериментальной, а в смеси аргон-ртуть с увеличением температуры $T$ до $70^{\circ} \mathrm{C}$ значение $U_{b}$ убывает, а при дальнейшем ее повышении возрастает, что обусловлено немонотонной зависимостью ИК смеси $\alpha$ от $n_{\mathrm{Hg}} / n$ (рис. 3 ) и согласуется с экспериментальными результатами $[10,12]$.

\section{Заключение}

В настоящей работе проведено моделирование кинетики основных типов заряженных и возбужденных частиц в слаботочном разряде в смеси аргон-ртуть, используемой в газоразрядных осветительных лампах. Рассчитанные зависимости ИК от приведенной напряженности электрического поля в чистых аргоне и ртути и их смеси, а также зависимость ИК смеси от относительного содержания ртути в ней, удовлетворительно согласуются с экспериметальными данными.

Найдены температурные зависимости вкладов различных механизмов образования и потерь ионов и метастабильных атомов в поддержание их баланса. Установлено, что основным механизмом образования атомарных ионов аргона является ионизация атомов аргона электронами, а атомарные ионы ртути образуются, главным образом, при пеннинговской ионизации атомов ртути метастабилями аргона. Потери же ионов обоих типов обусловлены преимущественно их дрейфом к катоду под действием электрического поля. Метастабили аргона и ртути образуются при возбуждении атомов аргона и ртути электронами, а разрушаются первые из них при пеннинговской ионизации атомов ртути, а вторые - в результате их тушения при столкновениях с невозбужденными атомами и диффузии к электродам. Влияние же всех других процессов на концентрации основных типов частиц, по крайней мере, на порядок меньше. Показано, что возрастание ИК в смеси аргон-ртуть по сравнению с чистым аргоном практически полностью обусловлено реакцией Пеннинга, причем ее роль увеличивается с уменьшением напряженности электрического поля в межэлектродном промежутке.

Рассчитаны также зависимости напряжения зажигания разряда от межэлектродного расстояния (кривые Пашена) при различных температурах смеси и дано объяснение немонотонной зависимости его величины от температуры.

Полученные результаты могут быть использованы для оценки влияния состава газовой смеси и эмиссионных свойств электродов на напряжение зажигания разряда в ртутных осветительных лампах. 
Работа выполнена в рамках Программы фундаментальных исследований НИУ ВШЭ, а также в рамках реализации государственного задания „Организация проведения научных исследований“ Минобрнауки РФ в МГТУ им. Н.Э. Баумана.

\section{Список литературы}

[1] Zissis G., Kitsinelis S. // J. Phys. D: Appl. Phys. 2009. Vol. 42. N 17. P. 173001.

[2] Samukawa S. et al. // J. Phys. D: Appl. Phys. 2012. Vol. 45. N 25. P. 253001.

[3] Schwieger J., Baumann B., Wolff M., Manders F., Suijker J. // J. Phys. Conf. Series. 2015. Vol. 655. P. 012045.

[4] Lister G.G., Lawler J.E., Lapatovich W.P., Godyak V.A. // Rev. Mod. Phys. 2004. Vol. 76. N 2. P. 541-598.

[5] Райзер Ю.П. Физика газового разряда. Долгопрудный: Издат. Дом Интеллект, 2009. 736 с.

[6] Кудрявиев А.А., Смирнов А.С., Цендин Л.Д. Физика тлеющего разряда. СПб.: Лань, 2010. 512 с.

[7] Byszewski W.W., Li Y.M., Budinger A.B., Gregor P.D. // Plasm. Sourc. Sci. Technol. 1996. Vol. 5. N 4. P. 720-735.

[8] Hadrath S., Beck M., Garner R.C., Lieder G., Ehlbeck J. // J. Phys. D: Appl. Phys. 2007. Vol. 40. N 1. P. 163-167.

[9] Brok W.J.M., Gendre M.F., van der Mullen J.J.A.M. // J. Phys. D: Appl. Phys. 2007. Vol. 40. N 1. P. 156-162.

[10] Sobota A., van den Bos R.A.J.M., Kroesen G., Manders F. // J. Appl. Phys. 2013. Vol. 113. N 4. P. 043308.

[11] Burgmans A.L.J., Smeets A.H.M. // J. Phys. D: Appl. Phys. 1983. Vol. 16. N 5. P. 755-762.

[12] Атаев A.E. Зажигание ртутных разрядных источников излучения высокого давления. М.: МЭИ, 1995. 168 с.

[13] Sawada S., Sakai Y., Tagashira H. // J. Phys. D: Appl. Phys. 1989. Vol. 22. N 2. P. 282-288.

[14] Миленин В.М., Тимофеев Н.А. Плазма газоразрядных источников света низкого давления. Л.: ЛГУ, 1991. 240 с.

[15] Zissis G., Bénétruy P., Bernat I. // Phys. Rev. A. 1992. Vol. 45. N 2. P. $1135-1148$.

[16] Bashlov N., Zissis G., Charrada K., Stambouli M., Milenin V., Timofeev N. // J. Phys. D: Appl. Phys. 1994. Vol. 27. N 3. P. 494-503.

[17] Petrov G.M., Giuliani J.L. // J. Appl. Phys. 2003. Vol. 94. N 1. P. 62-75.

[18] Ben Hamida M.B., Helali H., Araoud Z., Charrada K. // Phys. Plasmas. 2011. Vol. 18. N 6. P. 063506.

[19] Lay B., Moss R.S., Rauf S., Kushner M.J. // Plasma Sourc. Sci. Technol. 2003. Vol. 12. N 1. P. 8-21.

[20] Мокров М.С., Райзер Ю.П. // ЖТФ. 2008. Т. 78. Вып. 4. C. $47-54$.

[21] Богданов Е.А., Кудрявцев А.А., Чирцов А.С. // ЖТФ. 2011. T. 81. Вып. 1. С. 59-64.

[22] Eylenceoğlu E., Rafatov I., Kudryavtsev A.A. // Phys. Plasmas. 2015. Vol. 22. N 1. P. 013509.

[23] Rockwood S.D. // Phys. Rev. A. 1973. Vol. 8. N 5. P. 2348 2358.

[24] Phelps A.V. http://jilawww.colorado.edu/ãvp/

[25] Hyman H.A. // Phys. Rev. A. 1979. Vol. 20. N 3. P. 855-859.

[26] Bogaerts A., Gijbels R. // Phys. Rev. A. 1995. Vol. 52. N 5. P. 3743-3751.

[27] Bogaerts A., Gijbels R. // J. Appl. Phys. 1999. Vol. 86. N 8. P. 4124-4133.
[28] Lymberopoulos D.P., Economou D.J. // J. Appl. Phys. 1993. Vol. 73. N 8. P. 3668-3679.

[29] Sakai Y., Sawada S., Tagashira H. // J. Phys. D: Appl. Phys. 1989. Vol. 22. N 2. P. 276-281.

[30] Wamsley R.C., Mitsuhashi K., Lawler J.E. // Phys. Rev. E. 1993. Vol. 47. N 5. P. 3540-3546.

[31] Biondi M.A. // Phys. Rev. 1953. Vol. 90. N 5. P. 730-737.

[32] Крюков Н.А., Пенкин Н.П., Редько Т.П. // Опт. и спектр. 1977. T. 42. № 1. C. 33-41.

[33] Schlie L.A., Jusinski L.E., Rathge R.D., Drummond D.L., Hamil R.A. // J. Appl. Phys. 1980. Vol. 51. N 6. P. $3137-$ 3143.

[34] Moody S.E., Center R.E. // J. Appl. Phys. 1984. Vol. 55. N 7. P. 2721-2725.

[35] Кристя В.И., Фишер М.Р. // Изв. РАН. Сер. физ. 2010. T. 74. № 2. C. 298-301.

[36] Кристя В.И., Фишер М.Р. // Изв. РАН. Сер. физ. 2012. T. 76. № 5. С. 673-677.

[37] Bondarenko G.G., Fisher M.R., Kristya V.I. // J. Phys. Conf. Ser. 2012. Vol. 406. P. 012031.

[38] Donkó Z. // Plasma Sourc. Sci. Technol. 2011. Vol. 20. N 2. P. 024001.

[39] Таблицы физических величин / Под ред. И.К. Кикоина. М.: Атомиздат, 1976. 1006 с.

[40] Chanin L.M., Biondi M.A. // Phys. Rev. 1957. Vol. 107. N 5. P. 1219-1221.

[41] Kucukarpaci H.N., Lucas J. // J. Phys. D: Appl. Phys. 1981. Vol. 14. N 11. P. 2001-2014.

[42] Garamoon A.A., Abdelhaleem A.S. // J. Phys. D: Appl. Phys. 1979. Vol. 12. N 12. P. 2181-2187.

[43] Lisovskiy V.A., Yakovin S.D., Yegorenkov V.D. // J. Phys. D: Appl. Phys. 2000. Vol. 33. N 21. P. 2722-2730. 